\title{
Single-pulse radio observations of the Galactic Center magnetar PSR J1745-2900
}

\author{
Zhen Yan ${ }^{* 1,7}$, Zhi-Qiang Shen ${ }^{1,7}$, Xin-Ji Wu ${ }^{2}$, R. N. Manchester ${ }^{3}$, P. Weltevrede ${ }^{4}$, \\ Ya-Jun $\mathrm{Wu}^{1,7}$, Rong-Bing Zhao ${ }^{1,7}$, Jian-Ping Yuan ${ }^{5,7}$, Ke-Jia Lee ${ }^{6}$, Qing-Yuan Fan ${ }^{1,7}$, \\ Xiao-Yu Hong ${ }^{1,7}$, Dong-Rong Jiang ${ }^{1,7}$, Bin Li ${ }^{1,7}$, Shi-Guang Liang ${ }^{1,7}$, Quan-Bao Ling ${ }^{1,7}$, \\ Qing-Hui Liu ${ }^{1,7}$, Zhi-Han Qian ${ }^{1,7}$, Xiu-Zhong Zhang ${ }^{1,7}$, Wei-Ye Zhong ${ }^{1,7}$, Shu-Hua Ye ${ }^{1,7}$
}

\begin{abstract}
In this paper, we report radio observations of the Galactic Center magnetar PSR J1745-2900 at six epochs between June and October, 2014. These observations were carried out using the new Shanghai Tian Ma Radio Telescope at a frequency of $8.6 \mathrm{GHz}$. Both the flux density and integrated profile of PSR J1745-2900 show dramatic changes from epoch to epoch showing that the pulsar was in its "erratic" phase. On MJD 56836, the flux density of this magnetar was about $8.7 \mathrm{mJy}$, which was ten times large than that reported at the time of discovery, enabling a single-pulse analysis. The emission is dominated by narrow "spiky" pulses which follow a log-normal distribution in peak flux density. From 1913 pulses, we detected 53 pulses whose peak flux density is ten times greater than that of the integrated profile. They are concentrated in pulse phase at the peaks of the integrated profile. The pulse widths at the $50 \%$ level of these bright pulses was between $0.2^{\circ}$ to $0.9^{\circ}$, much narrower than that of integrated profile $\left(\sim 12^{\circ}\right)$. The observed pulse widths may be limited by interstellar scattering. No clear correlation was found between the widths and peak flux density of these pulses and no evidence was found for subpulse drifting. Relatively strong spiky pulses are also detected in the other five epochs of
\end{abstract}

\footnotetext{
${ }^{1}$ Shanghai Astronomical Observatory, Chinese Academy of Sciences, Shanghai 200030, China

${ }^{2}$ Department of Astronomy, Peking University, Beijing 100871, China

${ }^{3}$ CSIRO Astronomy and Space Science, PO Box 76, Epping NSW 1710, Australia

${ }^{4}$ Jodrell Bank Centre for Astrophysics, School of Physics and Astronomy, University of Manchester, Manchester M13 9PL, UK

${ }^{5}$ Xinjiang Astronomical Observatory, Chinese Academy of Sciences, Urumqi 830011, China

${ }^{6}$ Kavli Institute for Astronomy and Astrophysics, Peking University, Beijing 100871, China

${ }^{7}$ Key Laboratory of Radio Astronomy, Chinese Academy of Sciences, China
} 
observation, showing the same properties as that detected in MJD 56836. These strong spiky pulses cannot be classified as "giant" pulses but are more closely related to normal pulse emission.

Subject headings: pulsars: individual(PSR J1745-2900)

\section{Introduction}

Observations of a pulsar closely orbiting Sgr A*, the supermassive black hole at the Galactic Center (GC), could provide a variety of important information about the pulsar itself, the black hole, the interstellar medium around the GC and, if close enough, relativistic orbital dynamics. But, experience has shown that radio searches for pulsars near the GC are really challenging. Many searches have been made (Kramer et al. 2000; Johnston et al. 2006; Deneva et al. 2009; Macquart et al. 2010; Eatough et al. 2013b) but no pulsars were discovered within a few arcmin of Sgr A*. The closest separations between pulsars discovered by these projects and $\mathrm{Sgr} \mathrm{A}^{*}$ are about 10-15 arcmin.

PSR J1745-2900, which was serendipitously discovered, is the only pulsar known within a few arcmin from Sgr $A^{*}$. It was first detected as an X-ray flare thought to be from Sgr A* by Swift (Kennea et al. 2013). Follow-up observations with the NuSTAR X-ray Observatory detected periodic intensity variations with a period of $3.76 \mathrm{~s}$ (Mori et al. 2013). It is confirmed as a magnetar by subsequent X-ray timing observations with NuStar and Swift (Kaspi et al. 2014). Unlike ordinary pulsars, most of which can only be detected in the radio band, very few magnetars have been confirmed with radio pulsations. Of the 28 magnetars and candidates previously known, only three of them have detectable radio pulsations (Olausen \& Kaspi 2014). The radio pulsations from PSR J1745-2900 were detected by several large radio telescopes, which makes it the fourth magnetar known with radio pulsations (e.g., Eatough et al. 2013a).

Astrometry measurements for PSR J1745-2900 with the VLBA indicate that its projected separation from $\operatorname{Sgr} \mathrm{A}^{*}$ is as small as $0.097 \mathrm{pc}$ (Bower et al. 2014), giving it the potential to contribute to the important studies mentioned above. PSR J1745-2900 shows a flat radio spectrum and a high degree of polarized emission, according to observations at frequencies ranging from 1.4 to $20 \mathrm{GHz}$. The derived dispersion measure (DM) is $1778 \pm 3 \mathrm{~cm}^{-3} \mathrm{pc}$

*yanzhen@shao.ac.cn 
(Eatough et al. 2013a), which makes it the highest-DM pulsar known. The Faraday Rotation Measure $(\mathrm{RM})$ of this pulsar is $-66960 \pm 50 \mathrm{rad} \mathrm{m}^{-2}$. This implies that the magnetic field strength is about $2.6 \mathrm{mG}$ at the radius of $0.12 \mathrm{pc}$ from $\mathrm{Sgr} \mathrm{A}^{*}$, which is dynamically important for the accretion of the black hole (Eatough et al. 2013a; Shannon \& Johnston 2013). The scatter-broadening time scale at $1 \mathrm{GHz}$ and the scatter-broadening spectral index fitted with multi-frequency observations are $1.3 \pm 0.2 \mathrm{~s}$ and $-3.8 \pm 0.2$ respectively (Spitler et al. 2014). This timescale is several orders of magnitude lower than the prediction of the NE2001 model (Cordes \& Lazio 2002). Judging from this result, scattering effects are not the main reason for lack of pulsar detections around Sgr A*. The intrinsic deficit in the ordinary pulsar population in this area is proposed to be the most likely reason for this (Dexter \& O'Leary 2014; Chennamangalam \& Lorimer 2014).

Eight months of $8.7 \mathrm{GHz}$ observations of PSR J1745-2900 with the Green Bank Telescope (GBT) between MJDs 56515 and 56845 (Lynch et al. 2014) identified two main periods of activity. The first is characterized by approximately 5.5 months (up to MJD 56726) of relatively stable evolution in radio flux density, rotation and profile shape, while in the second period these properties became highly variable. The GBT observations gave a single-pulse energy distribution that roughly follows a log-normal distribution with an apparent high-energy tail. Subpulse drifting was not found in their observations. No sustained enhancement of the

X-ray emission at the GC was detected by the Swift on MJD 56910 (Degenaar et al. 2014). But, at present it is not clear that this X-ray enhancement is related to PSR J1745-2900 or Sgr A*.

In this paper, we present the results of $8.6 \mathrm{GHz}$ observations that were carried out with the Shanghai Tian Ma Radio Telescope (TMRT). The observation and data reduction procedures are presented in $\$ 2$. In $\$ 3$ and $\$ 4$ we discuss the integrated profile and single-pulse properties, respectively, and give further discussion about the results in $₫ 5$.

\section{Observations and data reduction procedures}

\subsection{Introduction to the TMRT}

The TMRT is a new $65 \mathrm{~m}$ diameter fully-steerable radio telescope located in the western suburbs of Shanghai, China. The first phase of construction was finished in December 2013. Four cryogenically cooled receivers covering the frequency ranges $1.25-1.75 \mathrm{GHz}, 2.2-$ $2.4 \mathrm{GHz}, 4.0-8.0 \mathrm{GHz}$ and $8.2-9.0 \mathrm{GHz}$, respectively, are available. The highest frequency of the TMRT will be $43 \mathrm{GHz}$. The telescope has an active surface control to compensate for gravity deformation of the main reflector during tracking. 
The digital backend system (DIBAS) of TMRT is an FPGA-based spectrometer based upon the design of Versatile GBT Astronomical Spectrometer (VEGAS) with pulsar modes that provide much the same capabilities as Green Bank Ultimate Pulsar Processing Instrument (GUPPI) (Ransom et al. 2009; Bussa 2012). For pulsar observations, DIBAS supports both pulsar searching and on-line folding mode. Both coherent and incoherent dedispersion observation modes are supported. For the incoherent dedispersion observation mode, the bandwidth of each of digitizer channel is up to $2 \mathrm{GHz}$. Since three pairs of digitizer (one digitizer for each polarization) are currently available, a maximum bandwidth of $6 \mathrm{GHz}$ can be supported. For the coherent dedispersion observation mode the maximum bandwidth is $1 \mathrm{GHz}$, limited by the computing power of the current high-performance computer cluster. DIBAS supports full Stokes-parameter pulsar observations which are written out in 8-bit PSRFITS format (Hotan et al. 2004)*. In order to reduce the data rate in pulsar searching observations, the observer can also choose to record Stokes-parameter I (total intensity) only, which is obtained by summing the two polarization channels after digitisation.

\subsection{Observations and data reduction}

Observations of PSR J1745-2900 were performed with the TMRT in 2014, June to October using the incoherent pulsar searching observation mode. The frequency range of our observations was $8.2-9.0 \mathrm{GHz}$. The full bandwidth was divided into 512 channels to allow off-line dedispersion. Even though the DM of PSR J1745-2900 is $1778 \mathrm{~cm}^{-3} \mathrm{pc}$, at this high frequency, 512 channels are sufficient, giving a time delay in each channel of only $\sim 46.9$ microsecond. The sampling interval was 131.07 microseconds. The exact observing dates were 2014 June 28, 2014 July 24, 2014 Aug. 4, 2014 Sept. 11, 2014 Sept. 12 and 2014 Oct. 13, corresponding to MJDs of 56836, 56862, 56873, 56911, 56912 and 56943, respectively. The data recording times for the six sessions were 120, 40, 25, 26, 60 and 90 min, respectively. The first two epochs of observation presented here occurred prior to the last epoch reported in Lynch et al. (2014).

The Digital Signal Processing for Pulsars (DSPSR) program (van Straten \& Bailes 2011) was used to dedisperse and fold the data at the known topocentric period using polynomial coefficients generated with TEMPO2 in its prediction mode (Hobbs et al. 2006). The profile data were written out with 1024 phase bins per period in PSRFITS format. The pulse broad-

ening caused by interstellar scattering is about 3.7 millisecond at $8.6 \mathrm{GHz}$ (Bower et al. 2014) and so it is reasonable to use 1024 phase bins across the 3.76 s pulse period. PSRCHIVE

*See also http://www.atnf.csiro.au/research/pulsar/index.html?n=Main.Psrfits 
programs (Hotan et al. 2004) were used to do further data editing and processing.

At the time of observation, the diode that injects pulsed signals into the front-end of the receiver had not been installed, so we could not use the normal procedure to do the flux density calibration. Previous studies have shown that the flux density of pulsars is in general intrinsically stable over several years. Most pulsars are in the weak scattering regime at $8.6 \mathrm{GHz}$ (Stinebring \& Condon 1990) and so it is reasonable to use other normal pulsars as calibrators to estimate the flux density of PSR J1745-2900. The pulsars chosen as the flux density calibrators were observed on the same day with PSR J1745-2900 using the same setups. There were no input power adjustments between observations of calibrators and PSR J1745-2900.

On MJD 56836, we observed five normal pulsars with known flux density at $8.35 \mathrm{GHz}$ with same setup as for PSR J1745-2900. Table 1 lists these pulsars and gives their $8.35 \mathrm{GHz}$ flux density measured at Effelsberg (Maron et al. 2013), radio spectral index (Malofeev et al. 1994), estimated flux density at $8.6 \mathrm{GHz}$, the flux density in arbitrary units measured with the TMRT, the average elevation angle of the TMRT during the observation, the length of the observation and the scaling factor to convert from TMRT units to mJy. Using the scaling factor of the five normal pulsars, we obtain the weighted average scaling factor and corresponding standard deviation. These are used to convert the measured flux density of PSR J1745-2900 into mJy units. The effect of possible variations in the flux of the known pulsars is negligible because of the stable intrinsic flux density of calibrator pulsars, weak interstellar scattering at the band of observation and the averaging effect of using multiple calibrators. Therefore, we only use the standard deviation of scaling factor to quantify the flux uncertainties of PSR J1745-2900.

Table 1: Flux densities of the calibration pulsars and of PSR J1745-2900 at MJD 56836

\begin{tabular}{lccccccc}
\hline Name & $\begin{array}{c}\mathrm{S}_{8.35} \\
(\mathrm{mJy})\end{array}$ & $\alpha$ & $\begin{array}{c}\mathrm{S}_{8.60} \\
\mathrm{mJy}\end{array}$ & $\begin{array}{c}\mathrm{S}_{\mathrm{TM}} \\
(\mathrm{arb})\end{array}$ & $\begin{array}{c}\text { Elev. } \\
\left({ }^{\circ}\right)\end{array}$ & $\begin{array}{c}\mathrm{T} \\
(\mathrm{min})\end{array}$ & Factor \\
\hline B0329+54 & $1.99 \pm 0.40$ & $3.8 \pm 0.9$ & $1.87 \pm 0.40$ & 0.00079768 & 49.8 & 120 & $2344 \pm 501$ \\
$\mathrm{~B} 0809+74$ & $0.64 \pm 0.13$ & $1.7 \pm 0.1$ & $0.61 \pm 0.13$ & 0.00024458 & 44.0 & 60 & $2494 \pm 532$ \\
$\mathrm{~B} 0823+26$ & $0.86 \pm 0.17$ & $1.8 \pm 0.1$ & $0.82 \pm 0.16$ & 0.00033217 & 49.7 & 60 & $2469 \pm 482$ \\
$\mathrm{~B} 0950+08$ & $1.09 \pm 0.22$ & $2.4 \pm 0.3$ & $1.02 \pm 0.21$ & 0.00051095 & 38.3 & 30 & $1996 \pm 410$ \\
$\mathrm{~B} 1133+16$ & $0.78 \pm 0.16$ & $2.0 \pm 0.1$ & $0.74 \pm 0.15$ & 0.00034609 & 41.5 & 90 & $2138 \pm 433$ \\
\hline J1745-2900 & - & - & $8.75 \pm 0.81$ & 0.00388361 & 29.3 & 120 & $2254 \pm 208$ \\
\hline
\end{tabular}

The estimated flux density of PSR J1745-2900 at $8.6 \mathrm{GHz}$ on MJD 56836 is $8.75 \pm$ $0.81 \mathrm{mJy}$. The GBT measurements carried out in the erratic phase of PSR J1745-2900 showed a mean flux density 11 mJy with a standard deviation of 8.5 mJy and mean uncertainties of ${ }_{-2.4}^{+3.1} \mathrm{mJy}$ (Lynch et al. 2014) and so the measured flux density of PSR J1745-2900 
is reasonable.

\section{Integrated profile of PSR J1745-2900}

The integrated profiles and phase-time plots of the region around the pulse for each PSR J1745-2900 TMRT observation are shown in Fig. 1, Clearly the pulse profile varies greatly over the span of the observations, although on the two adjacent days (MJDs 56911 and 56912) the profiles are the same within the noise.

For the other epochs of PSR J1745-2900 observation, at least two of calibrator pulsars listed in Table 1 were observed with the same observation setup. We obtain flux densities for PSR J1745-2900 on MJDs 56862, 56873, 56911, 56912 and 56943 of 2.69 $\pm 0.81,3.02 \pm 1.39$, $0.63 \pm 0.20,0.83 \pm 0.26$ and $1.83 \pm 0.35 \mathrm{mJy}$, respectively. For comparison, the flux density of this magnetar at the time of its discovery is only $0.2-0.8 \mathrm{mJy}$ (Eatough et al. 2013a). This variation cannot result from diffractive interstellar scintillation as the characteristic bandwidth is only of order $\mathrm{kHz}$ (Spitler et al. 2014) and so the observations average over many scintles. The timescale for refractive scintillation is of order years and hence it is possible that this could contribute to long-term intensity variations. However, the associated pulse profile changes (Fig. (1) must be intrinsic and it is probable that the intensity variations are also largely intrinsic. Lynch et al. (2014) found that the radio emission became stronger and more erratic from around MJD 56726 and our observations show that this erratic phase continued till at least MJD 56943. An enhancement of the X-ray emission at the GC was detected by Swift on MJD 56910 (Degenaar et al. 2014), but we found no obvious change in our radio observations of PSR J1745-2900 on two subsequent days (MJD 56911, 56912). This X-ray flare is probably associated with Sgr A*.

The integrated profile on MJD 56836 shown in Fig. 1 is comparable with that seen in GBT observations on MJDs 56751, 56794 and 56808, with a three-peak structure and precursor component. The precursor component was also detected in the integrated profile of this magnetar when it was discovered during its X-ray burst phase (around MJD 56410). But, at that time the main pulse was a single peak, not three peaks (Eatough et al. 2013a). The third component frequently showing in the GBT observations since MJD 56726 was also detected in our observations on MJDs 56836, 56862, 56873, 56911 and 56912. All of these observations suggest that the magnetar was in its erratic state during our observations. Because we only have six epochs of observation, it is impossible for us to update the pulsar timing parameters of this magnetar. In this paper, we focus on the single-pulse properties of this magnetar. 
The phase-time plots in Fig. 1 clearly show single-pulse emission spikes located within the phase window of the integrated profiles. These are more obvious in the plot corresponding to the MJD 56836 observation because of high signal-to-noise ratio $(\mathrm{S} / \mathrm{N})$ of this observation. Such spiky emission is not common in phase-time plots of other pulsars. In the following section we further discuss the single-pulse properties of PSR J1745-2900.

\section{Single pulses of PSR J1745-2900}

Normally, the system equivalent flux density (SEFD) of TMRT is about $50 \mathrm{Jy}$ at 8.6 GHz. In our observation, the integration time of each phase bin is $3.65 \mathrm{~ms}$; therefore only pulses whose peak exceeds about $30 \mathrm{mJy}$ will be detected at the $1 \sigma$ level. Since the duty cycle of J1745-2900 is less than $10 \%$, it is possible to detect single pulses in the MJD 56836 PSR J1745-2900 observation when its mean flux density was about 8.75 mJy. But, for other five epochs of observation, it is hard to detect most of individual pulses except some strong spiky pulses because of the limited $\mathrm{S} / \mathrm{N}$.

Fig. 2] shows two contiguous series of individual pulses from the observation of MJD 56836. From one period to the next, the positions of the narrow spikes appear to change randomly. However, within a given pulse the spikes appear to be quasi-periodic. PSR J1745-2900 observation with VLA on MJD 56486 indicated that these spikes have a typical separation of $\sim 10 \mathrm{~ms}$ (cf., Bower et al. 2014). This is discussed further below in $\$ 4.2$. Even though the spiky pulses are very narrow compared to the integrated pulse profile, there is evidence in Fig. 2 that some spikes have structure with two or more peaks or components.

\subsection{The peak flux density distribution of single pulses}

Here we present a statistical analysis of the energy distribution of single pulses based on the observation of MJD 56836. Following Lynch et al. (2014), we normalize the peak flux density in a given pulse, $S_{\mathrm{pk}}$, with the peak flux density of the integrated profile for the whole observation, $S_{\text {int,pk }}$. The histogram of the distribution of peak flux densities of all the 1913 pulses is shown in Fig. 3. It appears that the observed distribution follows a log-normal distribution:

$$
P_{\mathrm{ln}}=\frac{S_{\mathrm{int}, \mathrm{pk}}}{\sqrt{2 \pi} \sigma S_{\mathrm{pk}}} \exp \left[-\left(\ln \frac{S_{\mathrm{pk}}}{S_{\mathrm{int}, \mathrm{pk}}}-\mu\right)^{2} /\left(2 \sigma^{2}\right)\right]
$$

where $S_{\mathrm{pk}}$ and $S_{\mathrm{int}, \mathrm{pk}}$ are the peak flux density of a single pulse and the integrated pulse profile, respectively, and $\mu$ and $\sigma$ are the logarithmic mean and the standard deviation of the distribution. A Kolmogorov-Smirnov hypothesis test (Lilliefore 1967) is used to check 
whether or not the observed distribution can be described as log-normal. The $p$-value of log-normality hypothesis test is about 0.07 , which is greater than the threshold value 0.05, indicating that the observed distribution is consistent with a log-normal distribution. The observation data are fitted to the log-normal model using a least-squares fitting method. The solid curve in Fig. 3 is the best-fitting log-normal distribution with $\mu=1.34 \pm 0.02$ and $\sigma=0.57 \pm 0.02$.

In our observation of MJD 56836 we detected 53 bright pulses with peak flux density $S_{\mathrm{pk}}$ at least ten times larger than the peak flux density of the integrated profile $S_{\mathrm{int}, \mathrm{pk}}$. In order to do further studies on these bright pulse and distinguish whether or not there are some different properties between these strong pulses compared to others of lower peak flux density, we also try to characterize the shape of the 1913 pulses with the Gaussian fitting method. We find that most of the peak components of these pulses can be fitted with one Gaussian component. The profile of a sample of the pulses whose peak flux density exceeds ten times that of the integrated profile is shown in Fig. 4, along with the corresponding Gaussian fitting results.

With the Gaussian fitting method, we get the width at 50\% of peak (W50) and fitted peak flux density of single pulses of PSR J1745-2900. Historical observations of giant pulses indicate that there is a correlation between the peak flux density and the width of giant pulses, with the stronger pulses having narrower pulse widths (Jenet et al. 1998; Majid et al. 2011). This phenomenon is predicted by the giant-pulse radiation model of intensity amplification by induced Compton scattering in the pulsar magnetosphere plasma (Petrova 2004). In order to investigate whether this relation exists in the strong pulses of PSR J1745-2900, we give a scatter plot of the fitted peak flux density $S_{\mathrm{pk}}$ and the W50 width in Fig. 5. From the plot, it is clear that the W50 of pulses whose peak flux density exceeds ten times that of integrated file are in the range of $0.2^{\circ}$ to $0.9^{\circ}$. For comparison, W50 for the integrated profile is about $12^{\circ}$. The W50 of pulses with lower peak flux density are in a comparable but somewhat wider range $\left(0.1^{\circ}\right.$ to $\left.1.9^{\circ}\right)$. However, there is no clear correlation between peak flux density and pulse width. Even though the peak flux density of these pulses is high, their pulse energy is not that large, as their pulse widths are much narrower than that of the integrated profile.

The distribution in pulse phase of the 53 strong pulses is presented in Fig. 6. This shows that the strong pulses are preferentially emitted at the phases of the peaks in the integrated profile. No strong pulses were detected at the phase of the weaker leading component.

Spiky pulses with peak flux density exceeding ten times that of the integrated profile are also found in the other five epochs of observation data on PSR J1745-2900. The number of bright pulses detected in the observation of MJD 56862, 56873, 56911, 56912 and 56943, 
is $9,7,5,16$ and 13 respectively. Most of these pulses can also be fitted with one Gaussian component with a typical width in a range of $0.2^{\circ}$ to $1.2^{\circ}$. They also occur at the pulse phases of the peaks of the integrated profile.

\subsection{Subpulse drifting}

Many pulsars exhibit drifting subpulses, that is, a progressive shift of subpulse phase in successive pulses. Subpulse drifting can be characterized by two numbers: the horizontal separation between adjacent subpulses in pulse longitude $(P 2)$ and the vertical separation between drift bands in pulse periods $(P 3)$. Subpulse drifting can be detected using an

auto-correlation analysis (Taylor et al. 1975) or by Fourier methods (Edwards \& Stappers 2003). Other useful diagnostics of subpulse modulation are the longitude-resolved standard deviation (LRSD) and the longitude-resolved modulation index (LRMI). Useful diagnostic plots from the Fourier methods are the two-dimensional fluctuation spectrum (2DFS) and the longitude-resolved fluctuation spectrum (LRFS) which can be used to characterize $P 2$ and P3, respectively (Weltevrede et al. 2006, 2007).

To investigate the subpulse modulation properties of PSR J1745-2900, we calculate the LRSD, LRMI, LRFS and 2DFS for the observation of MJD 56835. In order to detect weak features and obtain information about the trends in an average sense, the input pulse series are divided into smaller blocks consisting of 256 pulses and so a fast Fourier transform length of 256 is used in our calculations. Each of these blocks was analyzed separately and the averaged results are shown in Fig. 7. The upper plot shows a strong pulse modulation with peaks in the modulation index at the phase of the peaks in the integrated profile. The middle panel shows that there are no strong periodicties in the subpulse modulation at any phase or in the phase-integrated spectrum. Likewise, the 2DFS in the lower plot shows no significant modulations in the vertical (pulse-to-pulse) direction, but there are significant broad peaks in the horizontal (pulse phase) direction. These correspond to the separation of the pulse components and the preferred phases of the strong pulses of about $8^{\circ}$ shown in Fig. 6, but do not represent a P2 modulation related to drifting subpulses.

\section{Discussion and conclusions}

Both the flux density and the integrated profile shape of PSR J1745-2900 show dramatic changes from epoch to epoch in our observations. This shows that the erratic phase starting around MJD 56726 seen in the GBT observations (Lynch et al. 2014) continued at least to 
our last observation on MJD 56943 (2014 Oct. 13). Dramatic changes in the flux density and integrated profile shape are also found for other magnetars whose radio radiation has been detected. Flux density variations observed in magnetar PSR J1550-5418 are up to $50 \%$ on timescales of a few days (Camilo et al. 2008). The flux density of magnetar PSR J1622-4950 varied by up to a factor of $\sim 10$ within a few days and, on average, decreased by a factor of 2 over 700 days (Levin et al. 2012). Clearly, the radio radiation of magnetars is not as stable as other radio pulsars. In normal pulsars the radio radiation is powered by the rotational energy loss, whereas in magnetars it is probably powered by the release of the magnetic-field energy. This difference in energy supply may account for the different nature of the emission.

To resolve the subpulse properties of PSR J1745-2900, observations must be made at relatively high radio frequencies to overcome the effects of interstellar scattering (Spitler et al. 2014). The TMRT observations reported in this paper were made in the band $8.2-9.0 \mathrm{GHz}$ with a nominal center frequency of $8.6 \mathrm{GHz}$. At these frequencies, the scattering time is of order a few ms. These observations, as well as previous observations at similar frequencies (Lynch et al. 2014; Bower et al. 2014), show that the subpulse structure is dominated by strong narrow spikes with a width of this order. The width of strong pulses in the TMRT observations ranged between $0.2^{\circ}$ and $0.9^{\circ}$, corresponding to approximately $2-9 \mathrm{~ms}$. It is possible that the observed pulse widths at frequencies around $8.6 \mathrm{GHz}$ are dominated by interstellar scattering and that the intrinsic widths of these strong pulses are narrower. In Fig. 4, there is some evidence for scattering tails on some pulses. The residuals between the observed pulses and Gaussian fitting results are often larger at the pulse tail; see, for example, pulses $\mathrm{N}=1279,1329$, 1387. Higher-frequency observations would be required to reveal true intrinsic pulse widths.

Analysis of the peak flux density distribution of the strong pulses relative to the peak of the integrated profile showed that it was well described by a log-normal distribution with mean in the logarithm of 1.34 and a standard deviation of 0.57 . There is no evidence for a high energy tail to the distribution. The GBT observations of Lynch et al. (2014) also showed a log-normal distribution with a similar standard deviation, but also showed a significant high-energy tail. This suggests that the pulse intensity distribution may be variable in time.

From the 1913 pulses observed on MJD 56836 we detected 53 pulses whose peak flux density exceeds ten times that of the integrated profile. No correlation was found between the width of the strong pulses and their peak flux density. Relatively bright spiky pulses were also detected in the other five epochs of our observation data with similar properties. The strong narrow pulses observed from PSR J1745-2900 are not sufficiently strong to be classed as "giant" pulses similar to those detected in some pulsars such as the Crab pulsar 
(Lundgren et al. 1995) and PSR B1937+21 (Kinkhabwala \& Thorsett 2000). Furthermore, in our observations at least, they do not have the power-law distribution in amplitude that characterizes giant pulses and they are not confined to a narrow phase range within the integrated profile.

For most normal pulsars, the single-pulse radio flux density distributions are also close to log-normal and most do not emit giant pulses (Cairns 2004; Burke-Spolaor et al. 2012). Only three magnetars currently have radio single-pulse observations and these give different results. Observations of PSR J1622-4950 at 3.1 GHz found that the flux density distribution followed a log-normal distribution (Levin et al. 2012). In contrast, simultaneous single-pulse observation of the radio emitting magnetar PSR J1809-1943 at frequencies of 1.4, 4.8 and 8.35 GHz by Serylak et al. (2009) found that its pulse-energy distributions were very peculiar, changing on a time scale of days and not able to be fitted by a single statistical model.

Bright spiky radio emission in the window of the integrated profile has been detected in a few normal pulsars, for example, PSRs B0656+14 (Weltevrede et al. 2006), B0943+10 (Backus et al. 2010) and PSR B0031-07 (Karuppusamy et al. 2011), and for two other magnetars, PSR J1809-1943 (Serylak et al. 2009) and PSR J1622-4950 (Levin et al. 2012). Strong magnetic fields at the light cylinder $B_{\mathrm{L}}$ and high spin-down luminosity $\dot{E}$ have been proposed as indicators of giant pulse emission (Cognard et al. 1996; Johnston \& Romani 2002; Knight 2006). In Table 2 we give $B_{\mathrm{L}}$ and $\dot{E}$ along with pulse period, characteristic age and surface dipole magnetic field for these six pulsars. Except for PSR B0031-07, these pulsars are comparatively young. The magnetic field at the light cylinder $B_{\mathrm{L}}$ and the spin-down luminosity $\dot{E}$ are not as high as for pulsars emitting giant pulses and are in the same range as other normal pulsars. This suggests that the strong spiky emission seen in magnetars and some normal pulsars is more closely related to normal radio emission and that the giant pulses have a different radiation mechanism.

Table 2: Parameters of pulsars with spiky radio emission

\begin{tabular}{lccccc}
\hline PSR & $\begin{array}{c}P_{0} \\
(\mathrm{~s})\end{array}$ & $\begin{array}{c}\tau_{\mathrm{C}} \\
(\mathrm{yr})\end{array}$ & $\begin{array}{c}\dot{E} \\
(\mathrm{ergs} / \mathrm{s})\end{array}$ & $\begin{array}{c}B_{\mathrm{S}} \\
(\mathrm{G})\end{array}$ & $\begin{array}{c}B_{\mathrm{L}} \\
(\mathrm{G})\end{array}$ \\
\hline B0031-07 & 0.94 & $3.66 \times 10^{7}$ & $1.92 \times 10^{31}$ & $6.27 \times 10^{11}$ & $7.02 \times 10^{0}$ \\
B0656+14 & 0.38 & $1.11 \times 10^{5}$ & $3.81 \times 10^{34}$ & $4.66 \times 10^{12}$ & $7.66 \times 10^{2}$ \\
B0943+10 & 1.10 & $4.98 \times 10^{6}$ & $1.04 \times 10^{32}$ & $1.98 \times 10^{12}$ & $1.40 \times 10^{1}$ \\
J1622-4950 & 4.33 & $4.03 \times 10^{3}$ & $8.29 \times 10^{33}$ & $2.74 \times 10^{14}$ & $3.18 \times 10^{1}$ \\
J1745-2900 & 3.76 & $4.31 \times 10^{4}$ & $1.02 \times 10^{33}$ & $7.30 \times 10^{13}$ & $1.28 \times 10^{1}$ \\
J1809-1943 & 5.54 & $1.13 \times 10^{4}$ & $1.80 \times 10^{33}$ & $2.10 \times 10^{14}$ & $1.16 \times 10^{1}$ \\
\hline
\end{tabular}




\section{Acknowledgments}

This work was supported in part by the National Natural Science Foundation of China (grants 11173046 and 11403073), Natural Science Foundation of Shanghai No. 13ZR1464500, National Basic Research Program of China (973 program) No. 2012CB821806, the Strategic Priority Research Program "The Emergence of Cosmological Structures" of the Chinese Academy of Sciences, Grant No. XDB09000000 and the Knowledge Innovation Program of the Chinese Academy of Sciences (Grant No. KJCX1-YW-18) and the Scientific Program of Shanghai Municipality (08DZ1160100).

\section{REFERENCES}

Backus, I., Mitra, D., \& Rankin, J. M. 2010, MNRAS, 404, 30

Bower, G. C., Deller, A., Demorest, P., et al. 2014, ApJ, 780, L2

Burke-Spolaor, S., Johnston, S., Bailes, M., et al. 2012, MNRAS, 423, 1351

Bussa, S. e. 2012, in American Astronomical Society Meeting Abstracts, Vol. 219, American Astronomical Society Meeting Abstracts \#219, 446.10

Cairns, I. H. 2004, ApJ, 610, 948

Camilo, F., Reynolds, J., Johnston, S., Halpern, J. P., \& Ransom, S. M. 2008, ApJ, 679, 681

Chennamangalam, J., \& Lorimer, D. R. 2014, MNRAS, 440, L86

Cognard, I., Shrauner, J. A., Taylor, J. H., \& Thorsett, S. E. 1996, ApJ, 457, 81

Cordes, J. M., \& Lazio, T. J. W. 2002, ArXiv Astrophysics e-prints, astro-ph/0207156

Degenaar, N., Reynolds, M., Miller, J., et al. 2014, The Astronomer's Telegram, 6458, 1

Deneva, J. S., Cordes, J. M., \& Lazio, T. J. W. 2009, ApJ, 702, L177

Dexter, J., \& O’Leary, R. M. 2014, ApJ, 783, L7

Eatough, R. P., Falcke, H., Karuppusamy, R., \& Lee, K. J. 2013a, Nature, 501, 391

Eatough, R. P., Kramer, M., Klein, B., et al. 2013b, in IAU Symposium, Vol. 291, IAU Symposium, ed. J. van Leeuwen, 382-384

Edwards, R. T., \& Stappers, B. W. 2003, A\&A, 407, 273 
Hobbs, G. B., Edwards, R. T., \& Manchester, R. N. 2006, MNRAS, 369, 655

Hotan, A. W., van Straten, W., \& Manchester, R. N. 2004, PASA, 21, 302

Jenet, F., Anderson, S., Kaspi, V., Prince, T., \& Unwin, S. 1998, ApJ, 498, 365

Johnston, S., Kramer, M., Lorimer, D. R., et al. 2006, MNRAS, 373, L6

Johnston, S., \& Romani, R. 2002, MNRAS, 332, 109

Karuppusamy, R., Stappers, B. W., \& Serylak, M. 2011, A\&A, 525, A55

Kaspi, V. M., Archibald, R. F., Bhalerao, V., et al. 2014, ApJ, 786, 84

Kennea, J. A., Burrows, D. N., Kouveliotou, C., et al. 2013, ApJ, 770, L24

Kinkhabwala, A., \& Thorsett, S. E. 2000, ApJ, 535, 365

Knight, H. S. 2006, 6, 41

Kramer, M., Klein, B., Lorimer, D., et al. 2000, in Astronomical Society of the Pacific Conference Series, Vol. 202, IAU Colloq. 177: Pulsar Astronomy - 2000 and Beyond, ed. M. Kramer, N. Wex, \& R. Wielebinski, 37

Levin, L., Bailes, M., Bates, S. D., et al. 2012, MNRAS, 422, 2489

Lilliefore, H. W. 1967, Journal of the American Statistical Association, 62, 399

Lundgren, S. C., Cordes, J. M., Ulmer, M., et al. 1995, ApJ, 453, 433

Lynch, R. S., Archibald, R. F., Kaspi, V. M., \& Scholz, P. 2015, ApJ, 806, 266

Macquart, J.-P., Kanekar, N., Frail, D. A., \& Ransom, S. M. 2010, ApJ, 715, 939

Majid, W. A., Naudet, C. J., Lowe, S. T., \& Kuiper, T. B. H. 2011, ApJ, 741, 53

Malofeev, V. M., Gil, J. A., Jessner, A., et al. 1994, A\&A, 285, 201

Maron, O., Serylak, M., Kijak, J., et al. 2013, A\&A, 555, A28

Mori, K., Gotthelf, E. V., Zhang, S., et al. 2013, ApJ, 770, L23

Olausen, S. A., \& Kaspi, V. M. 2014, ApJ, 212, 6

Petrova, S. A. 2004, A\&A, 424, 227 
Ransom, S. M., Demorest, P., Ford, J., et al. 2009, in American Astronomical Society Meeting Abstracts, Vol. 214, American Astronomical Society Meeting Abstracts, 605.08

Serylak, M., Stappers, B. W., Weltevrede, P., et al. 2009, MNRAS, 394, 295

Shannon, R. M., \& Johnston, S. 2013, MNRAS, 435, L29

Spitler, L. G., Lee, K. J., Eatough, R. P., et al. 2014, ApJ, 780, L3

Stinebring, D. R., \& Condon, J. J. 1990, ApJ, 352, 207

Taylor, J. H., Manchester, R. N., \& Huguenin, G. R. 1975, ApJ, 195, 513

van Straten, W., \& Bailes, M. 2011, PASA, 28, 1

Weltevrede, P., Edwards, R. T., \& Stappers, B. W. 2006, A\&A, 445, 243

Weltevrede, P., Stappers, B. W., \& Edwards, R. T. 2007, A\&A, 469, 607

Weltevrede, P., Stappers, B. W., Rankin, J. M., \& Wright, G. A. E. 2006, ApJ, 645, L149 


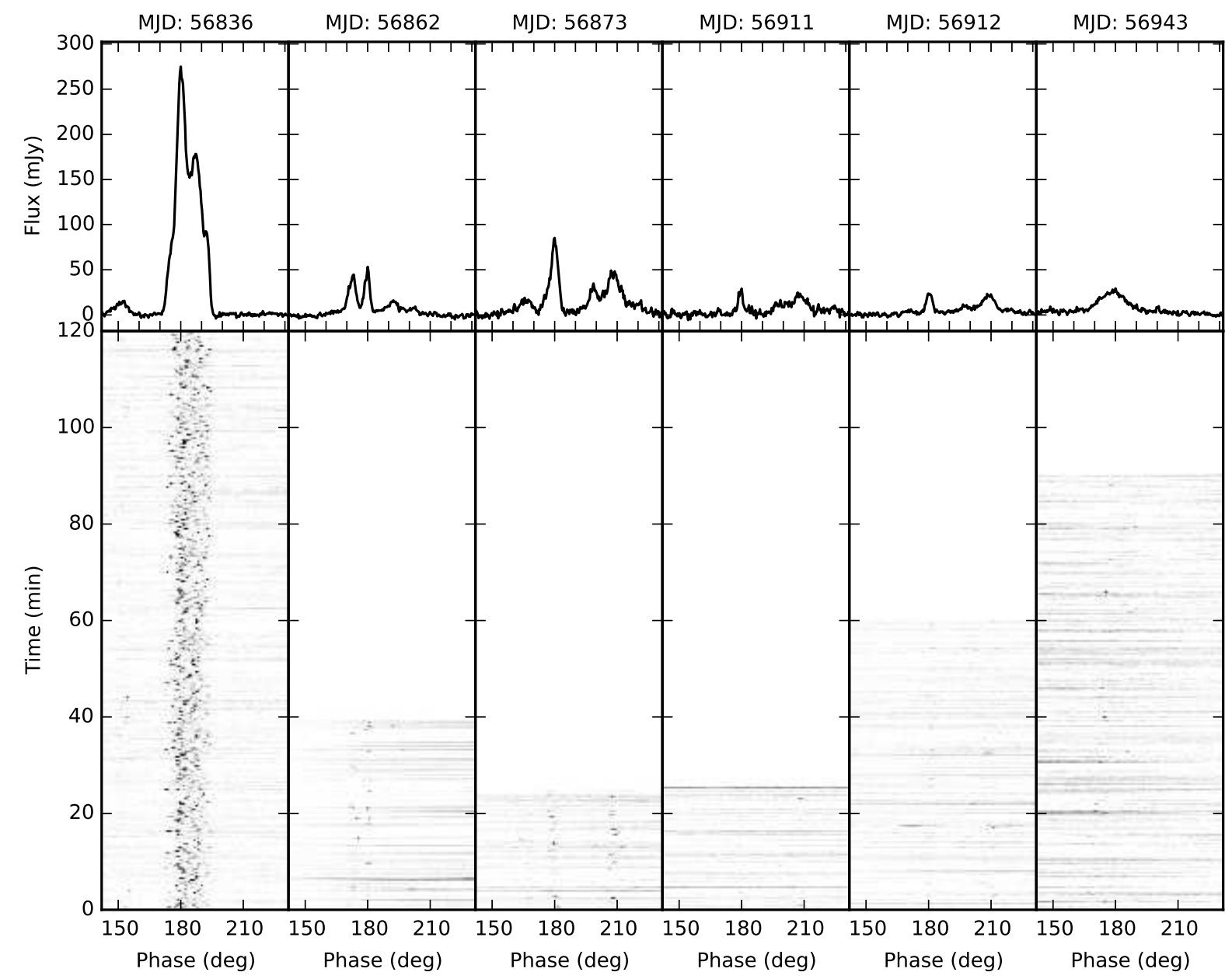

Fig. 1.- Integrated profiles and the phase-time plots of the magnetar PSR J1745-2900 on the six days of TMRT observations. The abscissa of each subplot is the rotational phase of the pulsar, with just the region around the pulse being shown. The MJD for the day of observation is given at the top of each subplot. All the plots use the same grayscale which is set according to the observations of MJD 56911 where the flux density is relatively low. The horizontal striping visible in the phase-time plots results from low-level radio-frequency interference (RFI). 

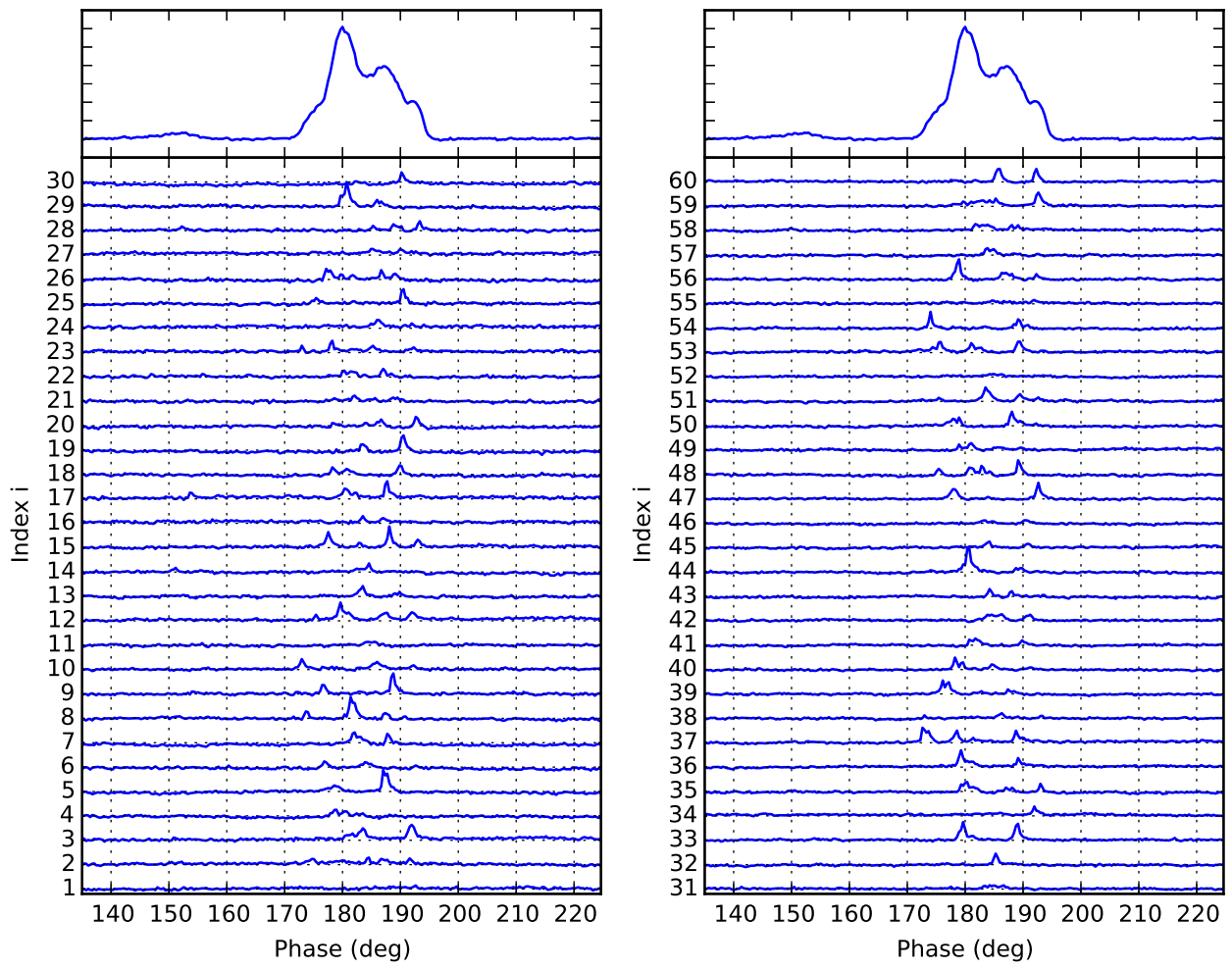

Fig. 2.- Single pulses from PSR J1745-2900 observed on MJD 56836. The actual pulse number counted from the start of the observation is $360+\mathrm{i}$. 


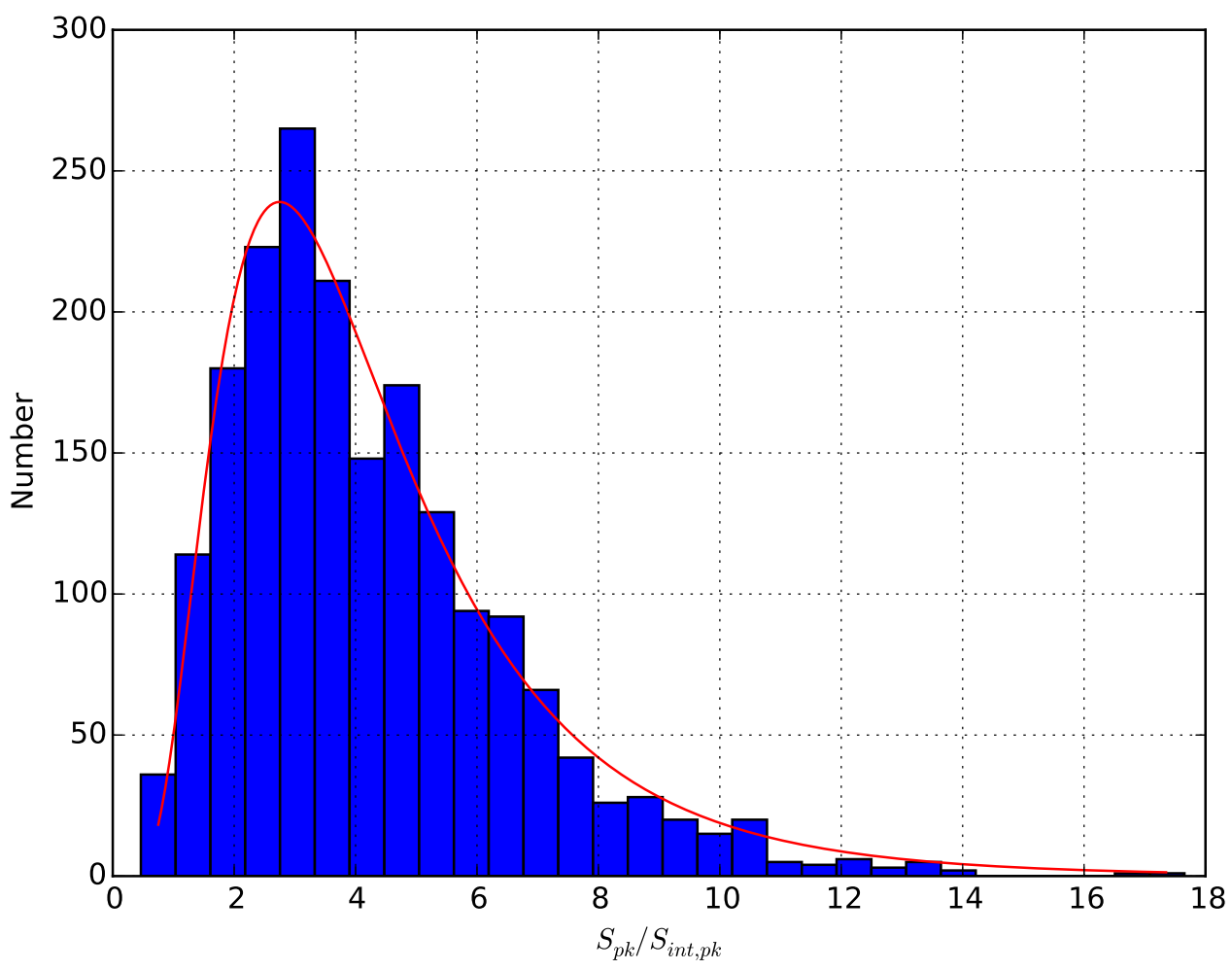

Fig. 3.- Distribution of the peak flux density of single pulses relative to the peak flux density of the mean pulse profile for the observation of MJD 56836. The best fitting lognormal function is shown as a solid curve. 

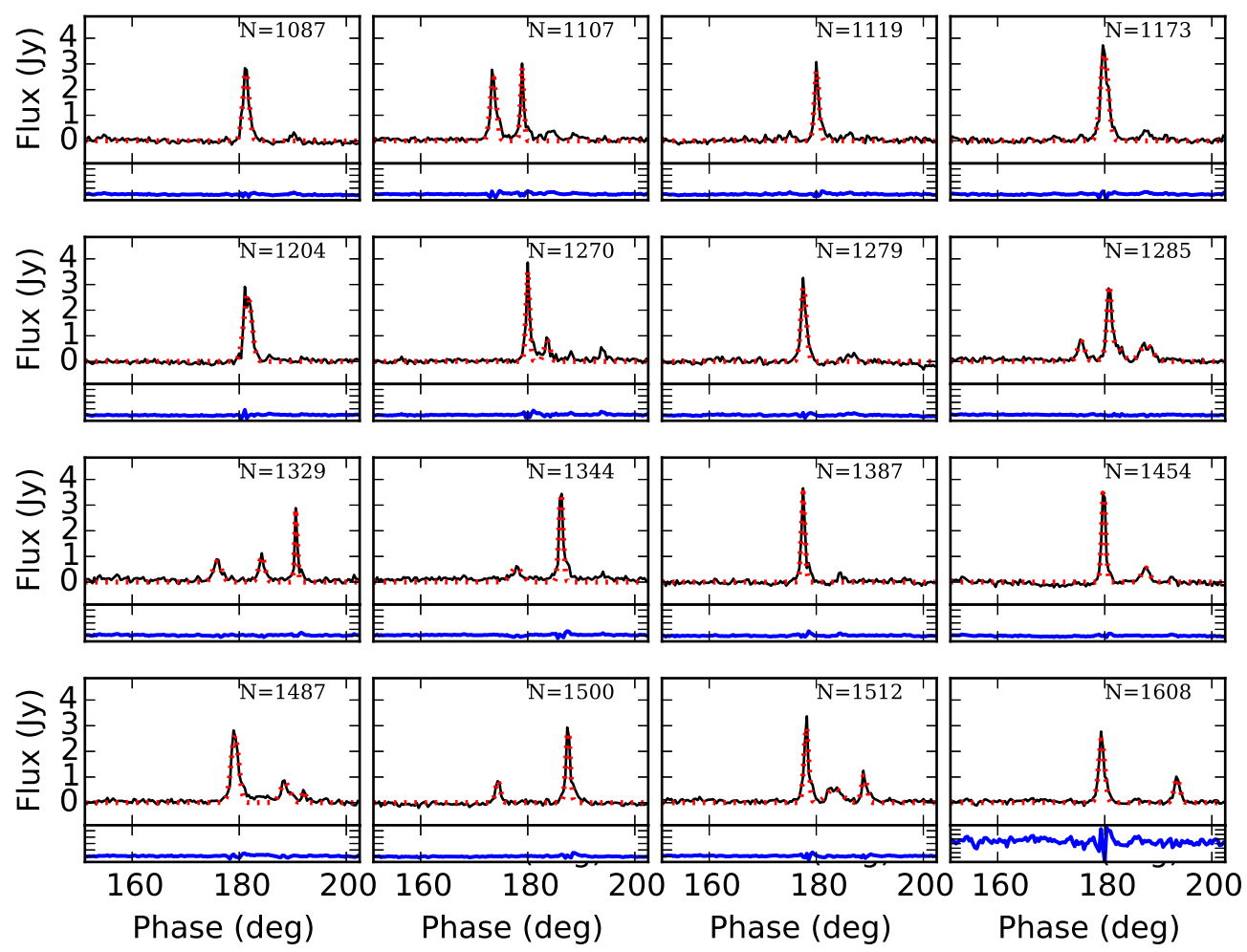

Fig. 4.- Profiles of pulses whose peak flux density exceeds ten times the peak flux density of the integrated profile. The observed pulsar profiles and fits of Gaussian profiles to the strong features are plotted as solid lines and the dotted lines, respectively. Pulse numbers are counted from the start of the observation. The residuals from the Gaussian fitting are shown at the bottom of each sub-panel. 


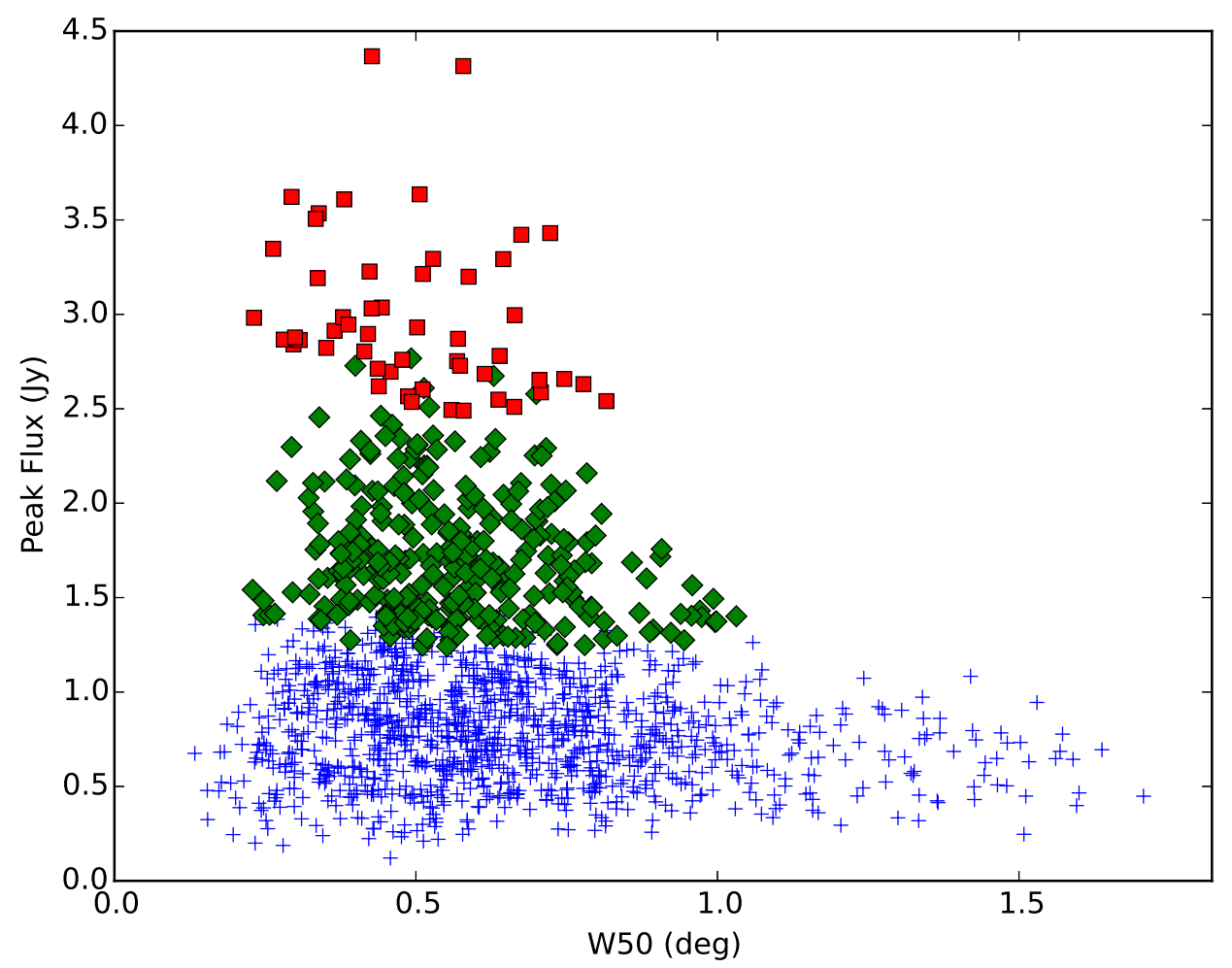

Fig. 5.- Peak flux density versus half-power width for the spikey pulses. Pulses whose $S_{\mathrm{pk}}$ is greater than 10 times $S_{\text {int,pk }}$, in the range of 5 to 10 and less than 5, are labelled with squares, diamonds and plus markers, respectively. 


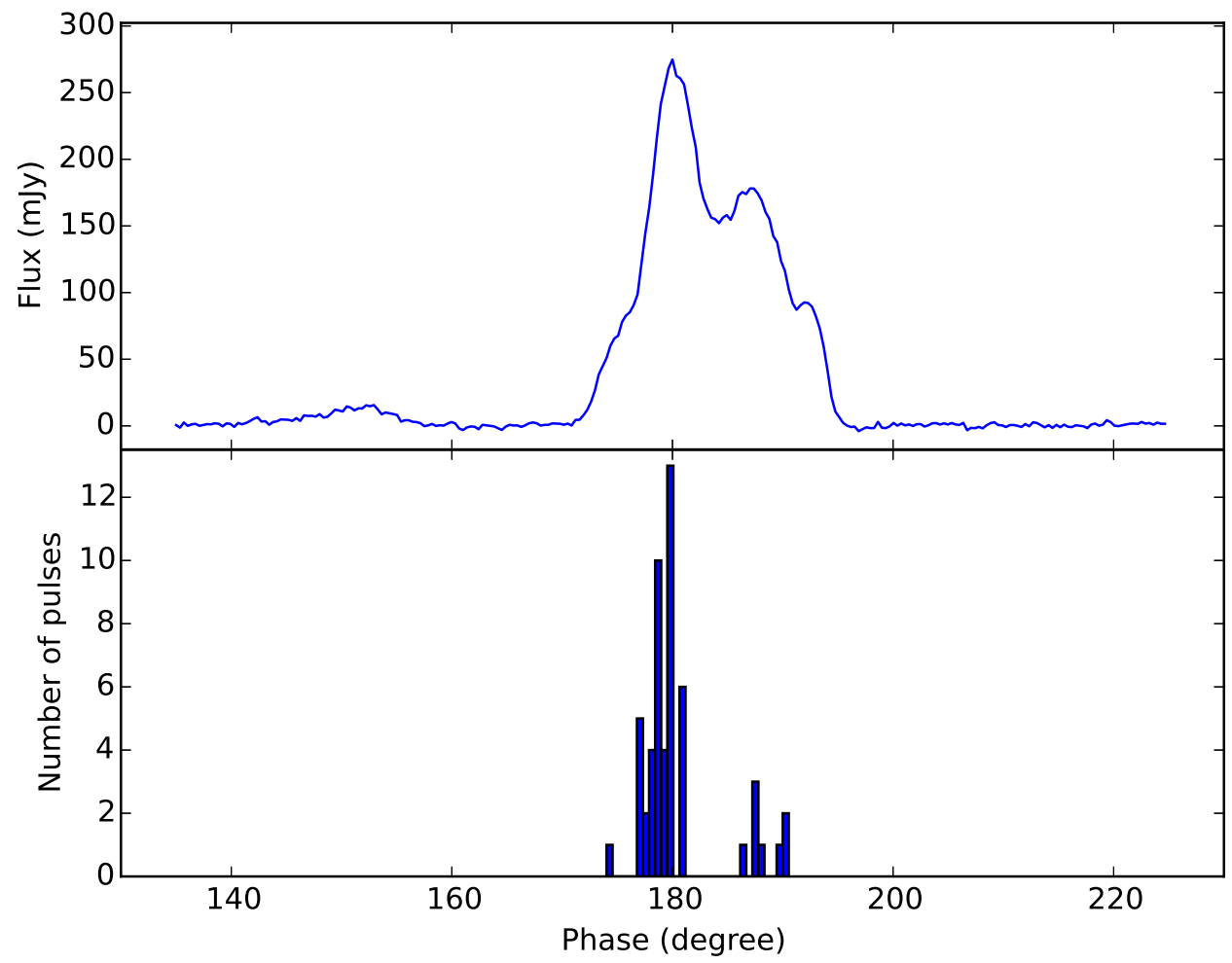

Fig. 6.- Histogram of central pulse phase for the 53 pulses with peak flux density greater than ten times the peak flux density of the integrated pulse profile. For comparison, the integrated pulse profile is shown in the top panel. 

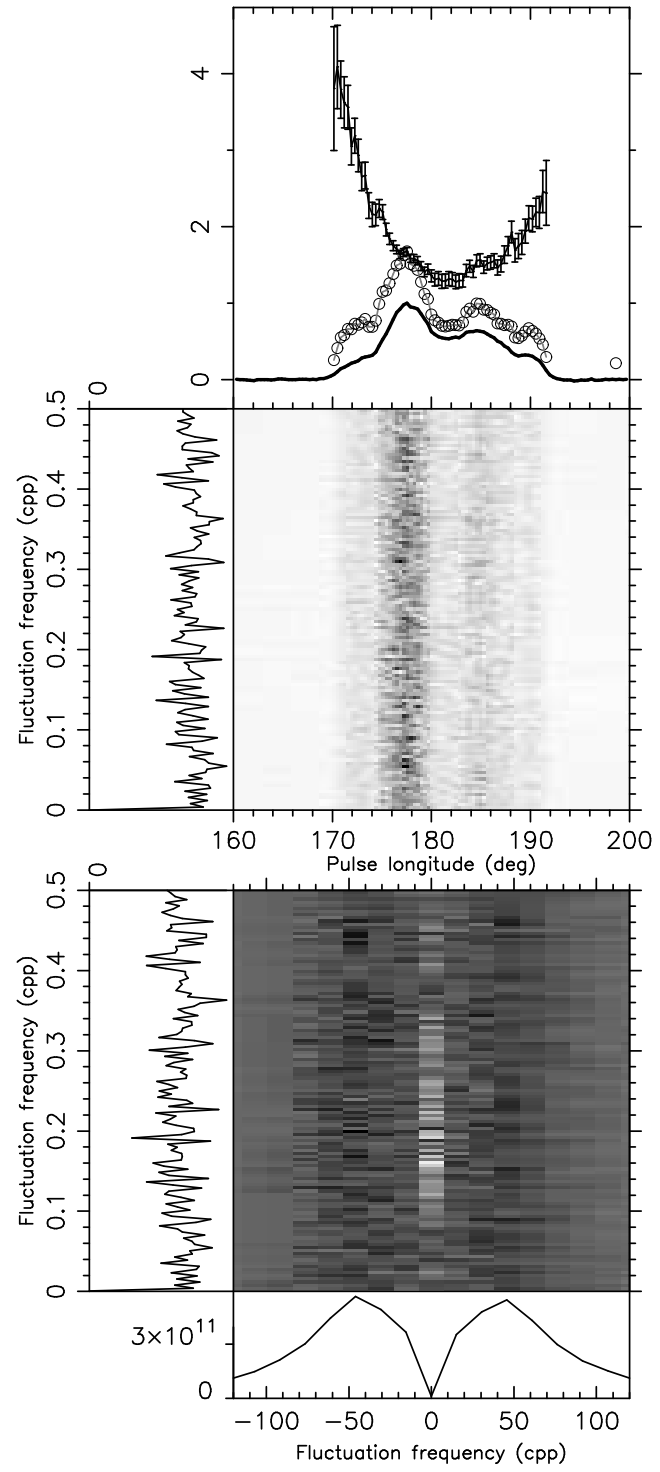

Fig. 7.- Results of subpulse analysis for the PSR J1745-2900 observation of MJD 56836. Upper panel: integrated pulse profile (solid line), longitude-resolved modulation index (error bars) and standard deviation (open circles). Middle panel: longitude-resolved fluctuation spectra with the phase-integrated spectrum on the left. Lower panel: two-dimensional fluctuation spectrum with integrated spectra on the left and below. 